\title{
Construcción y Validación de la Escala de Ansiedad ante el Desconfinamiento en Personas Expuestas a la Pandemia de COVID-19
}

\author{
Construction and Validation of the Disconfinement Anxiety Scale in People Exposed \\ to the COVID-19 Pandemic
}

\author{
Felipe E. García ${ }^{1}$, Mariela Andrades ${ }^{2}$ y Pedro Salinas ${ }^{3}$
}

\begin{abstract}
Resumen
La pandemia por Covid-19 ha obligado a confinar a la población por tiempos prolongados. La posibilidad de salir del hogar tras la cuarentena, puede producir ansiedad y miedo al contagio. El objetivo del presente estudio es construir un instrumento para evaluar la ansiedad por desconfinamiento y probar sus propiedades psicométricas. La Escala de Ansiedad ante el Desconfinamiento se aplicó en forma online a 417 personas de 18 años o más, hombres y mujeres, residentes en Chile. Se realizó un análisis factorial exploratorio que encontró dos dimensiones: evitación del contagio y malestar emocional. La escala mostró una correlación positiva con malestar general y negativa con satisfacción con la vida. La escala total obtuvo un $\alpha$ de Cronbach de .92 y sus subfactores explican una varianza de $51.24 \%$ del constructo. Estos resultados permiten contar con un instrumento válido y confiable para evaluar la ansiedad por desconfinamiento, que puede ser utilizado para detectar a personas vulnerables a problemas de salud mental durante o después de superada la pandemia.
\end{abstract}

Palabras clave: ansiedad, coronavirus, desconfinamiento, cuarentena, pandemia, salud mental

\begin{abstract}
The Covid-19 pandemic has forced the confinement of the population for prolonged periods of time. The possibility of leaving home after quarantine can produce anxiety and fear of contagion. The aim of the present study was to construct an instrument to assess the anxiety of being unconfined and to test its psychometric properties. The Disconfinement Anxiety Scale was administered online to 417 adults aged 18 years or older, men and women, living in Chile. An exploratory factor analysis was performed and found two dimensions: contagion avoidance and emotional distress. The scale showed a positive correlation with general distress and a negative correlation with satisfaction with life. The total scale obtained a Cronbach's $\alpha$ of .92 and its subfactors explain $51.24 \%$ of the total variance. These results present a valid and reliable instrument to assess disconfinement anxiety, which can be used to detect people vulnerable to mental health problems during or after the pandemic.
\end{abstract}

Keywords: anxiety, coronavirus, disconfinement, quarantine, pandemic, mental health

\footnotetext{
${ }^{1}$ Doctor en Psicología. Director Doctorado en Salud Mental. Departamento de Psiquiatría y Salud Mental, Facultad de Medicina. Programa de Neurociencia, Psiquiatría y Salud Mental NEPSAM. Universidad de Concepción, Chile. Correo: fgarciam@udec.cl (Autor de correspondencia).

${ }^{2}$ Doctora en Psicología. Académica Escuela de Psicología y Terapia Ocupacional, Universidad Central, Chile.

${ }^{3}$ Doctor en Psicología. Académico Instituto de Ciencias de la Facultad de Salud, Universidad Central, Chile. 


\section{Introducción}

El 31 de diciembre de 2019, la Organización Mundial de la Salud (OMS, 2020) tomó conocimiento de una nueva neumonía viral, originada en Wuhan, China. El 11 de marzo de 2020 la OMS declara la pandemia a nivel mundial por COVID-19. Desde entonces, y debido a la propagación del virus, han surgido estrictas medidas de confinamiento en gran parte del mundo, lo que ha llevado a cambios en la vida cotidiana, sin precedentes. El riesgo de contagio ha modificado la rutina diaria, el trabajo, los estudios, el estilo de vida y la economía del mundo (Gautam \& Sharma, 2020).

A lo largo de la historia, las enfermedades infecciosas han desencadenado miedo y ansiedad intensificados, afectando el bienestar psicológico y modificando los comportamiento de la población (Balaratnasingam \& Janca, 2006). En la actual pandemia por COVID-19 se ha observado un aumento en la ansiedad, depresión, estrés y síntomas de estrés postraumático (Talevi et al., 2020; Vindegaard \& Benros, 2020). Un estudio con personas altamente susceptibles a la infección por coronavirus, arrojó una tasa de prevalencia de estrés post-traumático de $73,4 \%$, depresión de $50,7 \%$ y ansiedad generalizada de $44,7 \%$ (Liu et al., 2020). En el caso particular de la ansiedad, un metaanálisis reciente reportó una prevalencia global del $31.9 \%$ entre 17 estudios publicados sobre este tema hasta marzo de 2020, con una muestra total de 63,439 participantes expuestos a la pandemia (Salari et al., 2020); un segundo meta-análisis reportó una prevalencia de $25 \%$ entre 43 estudios, lo que es un aumento considerable en relación a la prevalencia de $7.3 \%$ de problemas de ansiedad en población general en tiempos normales (Santabárbara et al., 2020).

Una de las medidas más drásticas tomadas en la actual pandemia ha sido el confinamiento obligatorio de gran parte de la población (Wang et al., 2020), pues si bien esto ha sido fundamental para mitigar la propagación de esta enfermedad, dicha medida está ocasionando serias consecuencias tanto a corto como a largo plazo (Galea et al., 2020). Ahora se ha especulado que no sólo el confinamiento provoca problemas en la salud mental, sino que también es posible que lo provoque el desconfinamiento, lo que hoy está ocurriendo en distintas partes del mundo de forma de no agotar a la población con largos encierros y poner de nuevo en movimiento las actividades productivas. Sin embargo, el desconfinamiento se realiza sin haber derrotado la pandemia, por lo que se mantiene el riesgo de contagio.

En Chile, por ejemplo, se inició la estrategia gradual para enfrentar la pandemia según la situación sanitaria de cada zona en particular. Se trata de cinco escenarios o pasos graduales con restricciones y obligaciones específicas, que van desde la cuarentena total obligatoria (nivel 1) hasta la completa liberación de las restricciones (nivel 5). De ese modo conviven en el mismo país localidades sin restricción, y kilómetros más allá, ciudades enteras bajo confinamiento. Dos veces a la semana, las autoridades sanitarias comunican al país las localidades que avanzan y las que retroceden en el proceso de desconfinamiento. Este plan, llamado Paso a Paso (Gobierno de Chile, 2020) implica los siguientes niveles, de los cuales el paso 5 no se ha aplicado hasta ahora en ninguna localidad debido a la mantención de la emergencia sanitaria: a) paso 1: confinamiento total, b) paso 2: cuarentena parcial los fines de semana, c) paso 3: se elimina la cuarentena y se retoman ciertas actividades con menor riesgo de contagio, con una cantidad de personas reducidas para mantener el distanciamiento social, d) paso 4: se permite aumentar la cantidad de personas en actividades habilitadas en la fase anterior, e) paso 5: apertura avanzada.

El progresivo desconfinamiento intra domiciliario supuso un imprevisto y complejo proceso para millones de personas en el mundo, marcado por un alto nivel de impredictibilidad y falta de control sobre las futuras condiciones de vida, dos variables que por sí solas son descritas en la literatura como altamente predisponentes en situaciones de estrés, ansiedad y depresión (Barlow et al., 2004). Por otra parte, el miedo y la ansiedad por infectarse con el coronavirus es una preocupación particular entre las personas que retoman sus actividades, especialmente las que podrían estar en contacto cercano con infectados con el virus (Ehrlich et al., 2020). Ceberio (2020) menciona que el miedo a contagiarse y la ansiedad ante el acercamiento físico, hace que las personas tiendan a retornar rápidamente al hogar después de salir al exterior. 
La ansiedad por desconfinamiento ha sido asociada por la prensa al "síndrome de la cabaña" (i.e.: Sepúlveda, 19 de julio de 2020). Si bien esta asociación es inexacta, pues originalmente el segundo término hacía referencia a la urgencia por abandonar el lugar de encierro aun conociendo el riesgo de hacerlo y que afectaba principalmente a los antiguos colonos americanos cuando tenían que pasar largas temporadas de invierno encerrados en cabañas (Ceberio, 2020), actualmente ha terminado aludiendo al malestar a la hora de abandonar el lugar donde una persona ha sido recluida durante un periodo largo de tiempo (Valdés, 2020). De ese modo, en el actual escenario de pandemia por coronavirus el término "síndrome de la cabaña" se ha popularizado para referirse a la ansiedad o miedo de salir del hogar cuando las autoridades sanitarias lo permitan, definición que incluso ha sido recogida por medios oficiales (Ministerio de Salud, 2021) e instituciones ligadas a la salud (Colegio Oficial de la Psicología de Madrid, 2020a)

Ceberio (2020) describe que es posible observar esta ansiedad en cierta ambivalencia con respecto a la salida de la cuarentena. Por una parte, existe el deseo de salir del encierro y, por otra, el temor a hacerlo. Esta paradoja de sentir malestar tanto por permanecer en el espacio físico como por salir de él podría producir una desregulación emocional, cognitiva y física (Prol \& Silva, 2020). Sus manifestaciones serían similares a la ansiedad general, con un alto malestar emocional y preocupación ante la inminencia de salir a la calle por el riesgo que supone hacerlo, y también tendría componentes del miedo, por lo que al malestar emocional se añadiría un comportamiento evitativo para reducir la posibilidad de contagio (Ceberio, 2020; Prol \& Silva, 2020).

En el contexto del presente estudio, se ha denominado a este malestar como ansiedad por desconfinamiento, considerándose un constructo vinculado a la ansiedad por COVID-19 y al miedo anticipatorio a ser contagiado o contagiar a un ser querido, y que tendría una fuerte relación con la ansiedad y el miedo como síntomas en general, pero que se trataría de constructos distintos, con un mayor nivel en quienes están actualmente en cuarentena total y en menor nivel en quienes viven en una localidad sin restricciones. De este modo, a partir de la pandemia muchas personas han evidenciado miedo a salir de casa o preocupación de que la próxima persona con la que entren en contacto pueda causarles daño (Sciarrino et al., 2020). Debido a ello, ha aumentado la ansiedad y miedo a salir de casa ya sea para trabajar (Silva, 2020) o para concurrir a centros de salud en caso que se necesite (Da et al., 2020; Wolf et al., 2020), lo que puede repercutir en una menor calidad de vida, por la pérdida de ingresos económicos o un empeoramiento en estados de salud.

El objetivo del presente estudio es desarrollar una escala para medir la ansiedad por desconfinamiento y mostrar sus propiedades psicométricas, específicamente su confiabilidad, validez de constructo, validez de criterio y validez concurrente. Además, se busca explorar el comportamiento de la variable en relación con características socio-demográficas y relativas al contagio por Covid-19. Se pretende evaluar principalmente su validez de constructo, lo que implica que debería contar de acuerdo a la literatura presentada con al menos dos dimensiones: un alto nivel de malestar emocional y comportamiento evitativo ante la posibilidad de salir de casa. También se esperan correlaciones moderadas con escalas de malestar emocional que pueden presentar las personas en forma concurrente. Se espera además que esta ansiedad por desconfinamiento sea más alta en quienes en el momento de la evaluación se encuentren en una localidad con cuarentena obligatoria (paso 1 en el plan paso a paso) y en menor nivel en quienes se encuentren en una localidad sin restricciones (paso 4 en el plan paso a paso).

Considerando el contexto actual, es relevante contar con instrumentos válidos y confiables que permitan a los profesionales de la salud mental explorar las reacciones de ansiedad en la población general y en los grupos más vulnerables y así, abordar el impacto de la pandemia en la salud mental de la población. Si bien existen escalas que miden ansiedad que se han utilizado en la pandemia, como la Escala de Ansiedad Generalizada (GAD-7) (Saravia et al., 2020) y la Escala de Depresión, Ansiedad y Estrés (DASS) (Andrades et al., 2021), ninguna de estas es específica a la ansiedad por desconfinamiento. Existe evidencia de al finalizar la cuarentena en 
pandemias anteriores, las personas aumentaron sus comportamientos de evitación afectando su calidad de vida (Marjanovic et al., 2007; Reynolds et al., 2008), por lo que contar con una escala de este tipo permitiría identificar casos probables de ansiedad disfuncional asociada con el coronavirus ante el desconfinamiento y tomar las medidas preventivas del caso.

\section{Método}

\section{Diseño}

Se utilizó un diseño instrumental, de tipo trasversal.

\section{Participantes}

Participaron 417 personas mayores de 18 años, de los cuales 332 fueron mujeres $(79.6 \%)$ y 85 hombres $(20.4 \%)$. La media total de edad fue de 35.01 años $(D T=12.90)$ con un rango desde los 18 a los 73 años. En relación a la etapa de desconfinamiento en la que estaba su ciudad de residencia al momento de contestar la encuesta según el plan paso a paso, un $54.1 \%$ estaba en etapa 1 , un $21.8 \%$ en la II, un $18.9 \%$ en la III y un $5.1 \%$ en la IV. Un $6 \%$ ha estado contagiado por Covid-19. Un 51.8\% tiene a un familiar, pareja o amigo cercano contagiado con Covid-19. Un $16.8 \%$ señala que un familiar, pareja o amigo cercano ha fallecido por Covid-19. Otros datos socio-demográficos se pueden observar en la Tabla 1.

Tabla 1. Características socio-demográficas de los participantes $(n=417)$

\begin{tabular}{llcc}
\hline Variable & Categorías & $\mathrm{N}$ & $\%$ \\
\hline Sexo & Masculino & 85 & 20.4 \\
& Femenino & 332 & 79.6 \\
Estado civil & Soltero & 248 & 59.5 \\
& Unión de hecho & 25 & 6 \\
& Casado & 101 & 24.2 \\
& Separado/divorciado & 39 & 9.4 \\
& Viudo & 4 & 1 \\
& & & \\
Nivel educacional & Básica & 1 & 0.2 \\
& Media & 35 & 8.4 \\
& Superior incompleta & 114 & 27.3 \\
& Superior completa & 162 & 38.8 \\
& Postgrado & 105 & 25.2 \\
\hline
\end{tabular}

\section{Instrumentos}

Escala de Ansiedad ante el Desconfinamiento (EAD), elaborado por los autores del presente artículo. El proceso de construcción de la escala se detalla en procedimiento. El Análisis Factorial Exploratorio (AFE) y otras propiedades psicométricas del instrumento se presentan en la sección de resultados.

Escala de Depresión, Ansiedad y Estrés (DASS-21) (Lovibond \& Lovibond, 1995), compuesta por 21 ítems agrupados en tres factores: Depresión, Ansiedad y Estrés, cada uno con siete ítems. Se responden en una escala Likert, que va desde 0 ("No describe nada de lo que me pasó o sentí en la semana") hasta 3 ("Sí, esto me pasó mucho, o casi siempre"). En su validación chilena (Antúnez \& Vinet, 2012), la escala obtuvo una confiabilidad satisfactoria, con valores $\alpha$ de .85 para Depresión, .73 para Ansiedad y de .83 para Estrés. Además, mostró validez de constructo al agruparse en el AFE en los tres factores propuestos.

Escala de Satisfacción con la Vida (SWLS), creada por Diener et al. (1985), en su versión para hablantes latinos de Arias y García (2018), la cual consta de cinco ítems tipo Likert, los cuales se responden en un rango que va desde 1 (totalmente en desacuerdo) a 7 (totalmente de acuerdo). En población latinoamericana ha mostrado indicadores de validez de constructo y concurrente y una consistencia interna de $\alpha=.81$ (Arias \& García, 2018).

Cuestionario de datos demográficos y datos relacionados con COVID-19. El cuestionario incluyó información relativa a sexo, edad, ciudad de residencia, situación de contagio con Covid-19 en ellos mismos o en alguien cercano $y$ fallecimiento de alguien cercano por Covid-19.

\section{Procedimiento}

Los ítems para construir la escala fueron elaborados a partir de la revisión bibliográfica de tipo narrativa sobre el miedo o ansiedad a salir del hogar tras periodos largos de confinamiento (i. e., Rosenblatt et al., 1984; Colegio Oficial de la Psicología de Madrid, 2020b) además de constructos relacionados como "ansiedad por covid" o "ansiedad por coronavirus" (Tan et al., 2020; Lee et al., 2020).

Se construyeron 45 ítems: 25 ítems desde diversos instrumentos ya elaborados sobre ansiedad por coronavirus y que fueron adaptados a la condición de encierro o confinamiento y 20 
ítems originales surgidos tras un proceso inductivo a posteriori que incluyó entrevistas semi-estructuradas a dos psicólogos clínicos y un psiquiatra especialistas en ansiedad y lectura de bibliografía relacionada.

Los ítems se sometieron al juicio de tres docentes universitarios expertos en psicometría. Solo fueron conservados los ítems para los que todos los jueces estuvieron de acuerdo sobre la relevancia del ítem, la congruencia con la definición de la dimensión medida (malestar emocional y comportamiento evitativo) y su pertinencia. Tras este proceso se eligieron 24 ítems.

Luego de la construcción de la escala se realizó un estudio piloto a 8 mujeres y 4 hombres de un rango de edad de 18 a 45 años, sin estudios superiores, para evaluar la comprensión de los ítems. El criterio para establecer el número de participantes en esta fase fue el de saturación de la información. Se descartaron cuatro ítems debido a que su redacción resultaba confusa para los participantes.

Tras este proceso, quedaron finalmente 20 ítems para la escala depurada que fue finalmente evaluada en el presente estudio.

La recolección de los datos se realizó vía online a través de un cuestionario autoadministrado construido con Google Forms, que se difundió mediante correos electrónicos, plataformas virtuales y redes sociales. Se buscó reclutar a un mínimo de 400 personas de público general, de 18 años o más, residentes en Chile. El tamaño muestral fue definido de acuerdo a las recomendaciones más exigentes para la realización de un $\mathrm{AFE}$, que señalan un número ideal de 300 a 400 participantes o de 20 participantes por ítem (Hair et al., 2010). El muestreo fue no probabilístico, por accesibilidad. La recopilación de datos se llevó a cabo desde el 14 de agosto hasta el 23 de agosto 2020.

Antes de responder el set de cuestionarios, los participantes debían leer una carta de consentimiento en el que se informó del carácter voluntario de la participación y de la confidencialidad de los datos, de acuerdo con las normas éticas vigentes para este tipo de estudios y según los cánones establecidos por la ley $\mathrm{N}^{\circ} 19.628$ de protección de datos de carácter personal en Chile. Luego de aceptar participar, pudieron acceder a los cuestionarios propiamente tal. Este estudio fue aprobado por el Comité de Ética de la Investigación Científica de la Universidad Central de Chile ( $N^{\circ} 18 / 2020$ ).

\section{Análisis de datos}

En primera instancia, se realizó un AFE de la Escala de Ansiedad ante el Desconfinamiento para evaluar su validez de constructo. Para determinar el número de factores se utilizó el análisis paralelo de Horn (O'Connor, 2000). Los ítems por factor se determinaron a través de la factorización de ejes principales con rotación Promax, debido a que teóricamente se esperan factores correlacionados, suprimiendo cargas factoriales inferiores a 40 .

Luego se calculó la correlación entre factores y la consistencia interna. A continuación se estableció la correlación con la Escala de Depresión, Ansiedad y Estrés y la Escala de Satisfacción con la Vida. Finalmente se realizaron comparaciones con variables incluidas en el cuestionario socio-demográfico.

Para todos estos análisis se utilizó el software estadístico SSPS Statistics 23.

\section{Resultados}

Se realiza AFE de la Escala de Ansiedad ante el Desconfinamiento, con los 20 ítems originales. El análisis paralelo determinó la existencia de dos factores. La factorización de ejes principales con rotación Promax permitió distinguir un ítem que no cargó en ningún factor (ítem 2: "La mayor parte de las personas tienen ganas de salir del encierro intradomiciliario, pero yo no me siento igual") y tres ítems (ítem 4: "Aun cuando las autoridades digan a futuro que es seguro salir, creo que preferiría no hacerlo"; ítem 6: "La mayor parte de las personas tienen ganas de salir del encierro intradomiciliario, pero yo no me siento igual"; ítem 11: "Cada vez que tengo que salir de mi casa me genera angustia") que cargaron en ambos, por lo que fueron eliminados.

Un nuevo análisis con estos cuatro ítems excluidos, volvió a distribuir los ítems en dos factores, mostrando coincidencias en el análisis paralelo, los valores propios superiores a $1 \mathrm{y}$ el gráfico de sedimentación, los que explican en total el $51.25 \%$ de la varianza. Los factores con sus ítems y cargas se observan en la Tabla 2. 
Tabla 2. Factor (varianza explicada) y cargas factoriales de la Escala de Ansiedad ante el Desconfinamiento $(n=417)$

\begin{tabular}{|c|c|c|}
\hline \multirow[b]{2}{*}{ Items } & \multicolumn{2}{|c|}{ Factor } \\
\hline & $\begin{array}{c}\text { Evitación } \\
\text { al contagio } \\
(43.84 \%)\end{array}$ & $\begin{array}{c}\text { Malestar } \\
\text { emocional } \\
(7.40 \%)\end{array}$ \\
\hline EAD 15: Evito salir a la calle por miedo a contagiarme & .78 & \\
\hline $\begin{array}{l}\text { EAD 20: Creo que lo mejor es quedarse en casa aun cuando digan que ya es seguro el retorno a la } \\
\text { normalidad }\end{array}$ & .74 & \\
\hline $\begin{array}{l}\text { EAD 14: Aun cuando las autoridades digan a futuro que es seguro retomar las actividades normalmente } \\
\text { fuera de casa siento miedo ante un posible contagio }\end{array}$ & .71 & \\
\hline EAD 17: Evito viajar en transporte público por un posible contagio & 67 & \\
\hline EAD 3: Pienso que estaré en riesgo al salir de mi casa y que las cosas podrían resultar mal & .66 & \\
\hline EAD 7: Aun cuando las autoridades digan a futuro que es seguro salir, creo que preferiría no hacerlo & .64 & \\
\hline EAD 8: Evito estar en filas, ir a supermercados y a todo lugar donde haya aglomeración de gente & 62 & \\
\hline $\begin{array}{l}\text { EAD 19: Creo que, aunque tome todas las precauciones igualmente se puede ver expuesta la salud tras el } \\
\text { desconfinamiento }\end{array}$ & .62 & \\
\hline EAD 16: Me da miedo retomar las relaciones sociales presenciales (ver a amigos, compañeros de trabajo) & .58 & \\
\hline EAD 1: Evito el contacto con la gente & .56 & \\
\hline EAD 12: Me da tristeza retomar mis actividades & & .97 \\
\hline EAD 18: Cuando pienso en retomar la vida qué tenía antes de la cuarentena, me da tristeza & & .87 \\
\hline EAD 9: El solo hecho de pensar en tener que retomar la vida normalmente me hace sentirme angustiado & & .81 \\
\hline EAD 13: Me he sentido temeroso/a de volver a retomar la vida con normalidad después de la pandemia & & .59 \\
\hline EAD 5: Ya no tengo tantas ganas de retomar la normalidad como lo sentía al comienzo de la cuarentena & & .57 \\
\hline EAD 11: Cada vez que tengo que salir de mi casa me genera angustia & & .42 \\
\hline
\end{tabular}

Nota. EAD=Escala de Ansiedad ante el Desconfinamiento

Tabla 3. Estadísticos descriptivos y correlaciones entre la Escala de Ansiedad ante el Desconfinamiento, la Escala de Depresión, Ansiedad y Estrés, y la Escala de Satisfacción Vital (n=417)

\begin{tabular}{|c|c|c|c|c|c|c|c|c|}
\hline & $\mathrm{M}$ & $\mathrm{DE}$ & 2 & 3 & 4 & 5 & 6 & 7 \\
\hline 1. Satisfacción con la vida & 24.66 & 5.83 & $-.53 * * *$ & $-.35 * * *$ & $-.31 * * *$ & $-.11 *$ & $-.18 * * *$ & $-.15 *$ \\
\hline 2. DASS Depresión & 5.98 & 5.27 & na & $.71 * * *$ & $.77 * * *$ & $.32 * * *$ & $.46 * * *$ & $.42 * * *$ \\
\hline 3. DASS Ansiedad & 5.29 & 5.02 & & na & $.75 * * *$ & $.33 * * *$ & $.45 * * *$ & $.42 * * *$ \\
\hline 4. DASS Estrés & 8.91 & 5.04 & & & na & $.40 * * *$ & $.46 * * *$ & $.47 * * *$ \\
\hline 5. EAD Evitación & 37.22 & 7.67 & & & & na & $.67 * * *$ & $.94 * * *$ \\
\hline 6. EAD Malestar & 17,47 & 5.82 & & & & & na & $.89 * * *$ \\
\hline 7. EAD Total & 54.69 & 12.34 & & & & & & na \\
\hline
\end{tabular}

DASS=Escala de Depresión, Ansiedad y Estrés; EAD=Escala de Ansiedad ante el Desconfinamiento; na=no aplica; * $p<.05 ; * * * p<.001$

El primer factor fue llamado Evitación al contagio. El factor 2 fue denominado Malestar emocional. Ambos factores obtuvieron una consistencia interna equivalente a un $\alpha=.88$, mientras que la escala total con los 16 ítems obtuvo un $\alpha=.92$. La correlación entre los dos factores fue de $r=.67$.

Para evaluar la validez concurrente de la EAD se midió su correlación con la Escala DASS y la escala de Satisfacción Vital, obteniéndose las relaciones que se observan en la Tabla 3.

En la Tabla 3 constatamos que la EAD correlaciona negativamente con la satisfacción con la vida $(r=-.15)$ y positivamente con las escalas de depresión, ansiedad y estrés ( $r=.42$ a .47).

$\mathrm{Al}$ analizar la relación de la EAD con la edad, se observa una correlación significativa solo con la evitación al contagio, en donde a mayor edad menor evitación ( $r=-.14 ; p=.005)$.
$\mathrm{Al}$ realizar comparaciones con otras variables socio-demográficas y relativas a la pandemia de tipo categóricas, utilizando para ello la $t$ de Student para muestras independientes, se observaron los resultados que se muestran en la Tabla 4.

En un último análisis, se realizó una comparación por etapa de desconfinamiento en la que se encuentra el lugar de residencia del participante al momento de contestar la encuesta. Para ello se realizó una prueba ANOVA de un factor. Se observa que quienes están en la etapa 1 manfiestan más ansiedad ante del desconfinamiento, tanto en la escala total, como en sus dos factores, que quienes están en las etapas 3 y 4 . En el factor de evitación se obtuvo un $F_{(3,408)}=3.113, p=.026$, donde quienes están en etapa 1 presentan puntajes más altos $(M=38.19$; $D E=7.73$ ) que quienes están en las etapas 3 
Tabla 4. Comparaciones t de Student en ansiedad ante el desconfinamiento y sus factores de evitación y malestar de acuerdo a características socio-demográficas $(\mathrm{n}=417)$

\begin{tabular}{|c|c|c|c|c|c|c|}
\hline \multirow{2}{*}{ Variable } & \multicolumn{2}{|c|}{ EAD evitación } & \multicolumn{2}{|c|}{ EAD malestar } & \multicolumn{2}{|c|}{ EAD total } \\
\hline & $M(D E)$ & valor $t$ & $M(D E)$ & valor $t$ & $M(D E)$ & valor $t$ \\
\hline \multicolumn{7}{|c|}{ Sexo } \\
\hline Mujer & $37.64(7.36)$ & $2.254 *$ & $18.08(5.70)$ & $4.309 * * *$ & $55.72(11.87)$ & $3.429 * * *$ \\
\hline Hombre & $35.55(8.64)$ & & $15.09(5.67)$ & & $50.65(13.34)$ & \\
\hline \multicolumn{7}{|c|}{ ¿Algún familiar, pareja o amigo cercano se ha contagiado de COVID-19? } \\
\hline No & $36.07(8.35)$ & $-2.956 * *$ & $17.07(5.91)$ & -1.340 & $53.14(13.13)$ & $-2.468 *$ \\
\hline Sí & $38.29(6.83)$ & & $17.84(5.72)$ & & $56.13(11.39)$ & \\
\hline \multicolumn{7}{|c|}{ ¿Algún familiar, pareja o amigo cercano ha fallecido de COVID-19? } \\
\hline No & $37,10(7.71)$ & -0.695 & $17.35(5.88)$ & -0.948 & $54.45(12.42)$ & -0.879 \\
\hline Sí & $37.80(7.51)$ & & $18.07(5.52)$ & & $55.87(11.91)$ & \\
\hline \multicolumn{7}{|c|}{ ¿Usted ha sido diagnosticado con Covid-19? } \\
\hline No & $37.14(7.73)$ & -0.821 & $17.40(5.89)$ & -1.228 & $54.54(12.47)$ & -0.966 \\
\hline Sí & $38.44(6.68)$ & & $18.56(4.48)$ & & $57.00(9.89)$ & \\
\hline
\end{tabular}

$* p<.05 ; * * p<.01 ; * * * p<.001$

$(M=35.83 ; D E=7.60)$ о $4(M=34.43 ; D E=8.27)$. En el factor de malestar se obtuvo un $F_{(3,408)}=2.800, p=.04$, donde quienes están en etapa 1 presentan puntajes más altos $(M=18.19$; $D E=5.95)$ que quienes están en las etapas 3 $(M=16.42 ; D E=5.78)$ о $4(M=15.81 ; D E=6.61)$. En la escala total se obtuvo un $F_{(3,408)}=3.554$, $p=.015$, donde quienes están en etapa 1 presentan puntajes más altos $(M=56.38 ; D E=12.41)$ que quienes están en las etapas $3 \quad(M=52.26$; $D E=12.26)$ o $4(M=50.24 ; D E=14.10)$.

Para determinar la capacidad de discriminación global de la EAD, se calcularon curvas ROC (Receiver Operating Characteristic) comparando con la subescala de ansiedad de la DASS que informa un puntaje de corte de 5 o más para un nivel de ansiedad leve o superior (Román et al., 2016). El Área Bajo la Curva (ABC) es superior a .70 indicando que la EAD es adecuada para detectar los síntomas que evalúa. De acuerdo a este análisis, se propone como puntaje de corte el valor de $52(>51)$, el cual tiene una sensibilidad de 72,30 y una especificidad de 45,50 .

\section{Discusión}

En la presente investigación se evaluaron las propiedades psicométricas de un instrumento elaborado para medir la ansiedad por desconfinamiento. El instrumento denominado Escala de Ansiedad ante el Desconfinamiento (EAD) mostró estar constituido por dos factores: evitación al contagio y malestar emocional.
La evitación es un componente central del miedo y la ansiedad y en el presente caso se orienta a la evitación de conductas que puedan aumentar el riesgo de contagio, como lugares cerrados o aglomerados (Reynolds et al., 2008). Esta ansiedad ante la probabilidad de un acercamiento físico no deseado, llevaría a las personas a querer retornar al hogar (Ceberio, 2020). Esto concuerdan con un estudio realizado a partir del desconfinamiento tras el brote del síndrome respiratorio agudo severo (SARS) en el año 2004, en el que luego de una cuarentena se observaron comportamientos de evitación, como la disminución del contacto directo con otras personas y no presentarse al trabajo (Marjanovic et al., 2007). Otro estudio con el mismo sindrome arrojó que el $54 \%$ de los participantes evitaba a personas que tosían o estornudaban, el $26 \%$, evitaba los lugares cerrados con mucha gente y el $21 \%$ evitaba todos los espacios en las semanas posteriores al período de cuarentena (Reynolds et al., 2008).

El segundo factor de malestar emocional refleja la naturaleza desagradable de la ansiedad por desconfinamiento, que si bien cumple con una función que permite proteger al individuo del riesgo de contagio (Sandín et al., 2020), de igual modo genera alteraciones a la vida cotidiana, pues la sociedad y la propia supervivencia requieren que las personas abandonen su hogar para trabajar, buscar suministros, realizar trámites y relacionarse socialmente, entre otras necesidades, por lo que este miedo a salir de casa puede ser 
percibido como una respuesta molesta y desadaptativa (Ceberio, 2020).

La EAD presentó las correlaciones hipotetizadas con la Escala de Depresión, Ansiedad y Estrés (DASS-21) y Escala de Satisfacción con la Vida (SWLS). Es decir, las correlaciones fueron directas con DASS e inversas con SWLS. Estas correlaciones con DASS no son altas por lo que se concluye que si bien la ansiedad por desconfinamiento va en la misma dirección del malestar general, incluyendo la ansiedad, se trataría de constructos distintos: el primero aludiendo a una ansiedad específica relacionado a la posibilidad de salir del hogar tras largo tiempo confinado ("El solo hecho de pensar en tener que retomar la vida normalmente me hace sentir angustiado", "Cada vez que tengo que salir de mi casa me genera angustia"), el segundo a una ansiedad de tipo más general ("Estaba preocupado por situaciones en las cuales podía tener pánico o en las que podría hacer el ridículo", "tuve miedo sin razón"). Así mismo, el miedo ante el desconfinamiento afectaría el bienestar de las personas afectadas.

En cuanto al sexo, las mujeres presentaron más ansiedad ante el desconfinamiento que los hombres. Esta diferencia ha estado presente en la mayoría de los estudios realizados en torno a la salud mental a partir de la pandemia (Li et al., 2020; Sandín et al., 2020). En relación a la ansiedad ante el deconfinamiento, una posible explicación es que las mujeres están preocupadas por su salud en general, tanto física como mental, debido a la pandemia, lo que las lleva a presentar un temor mayor que los hombres (Dagnino et al., 2020). Así mismo, es posible hipotetizar que los hombres presentan menor ansiedad que las mujeres debido a una cierta tendencia a minimizar el riesgo, tal como indican estudios interconductuales en torno a la percepción de riesgo (Martínez-Munguía et al., 2014)

En cuanto a la relación con la edad, en la presente investigación se observó que las personas aumentaban su evitación a medida que tenían menor edad. Andrades et al (2021) realizaron un estudio con población chilena al inicio de la pandemia, encontrando también que las personas de menor edad presentaban mayores niveles de malestar emocional que las personas de mayor edad, tal como también se encontró en España
(Sandín et al., 2020). La explicación planteada por Andrades et al (2021) fue que para las personas más jóvenes esta pandemia ha significado una amenaza importante para sus proyectos de vida, como el comenzar o terminar una carrera profesional, y para sus actividades más cotidianas como la vida social con pares o el desarrollo de actividad física, muy relevantes en este periodo de la vida. Bao et al. (2020) agregan que los jóvenes buscan información a través de sus redes sociales, por lo que están más expuestos a información falsa que les genera alarma. Es probable que estas sean algunas de las razones por la que finalmente exponerse al contagio sea algo que deseen evitar.

Otro resultado señala que la existencia de un familiar diagnosticado con COVID-19, aumenta la ansiedad ante el desconfinamiento. Esto podría relacionarse con el miedo al contagio, a que se propague el virus y al temor a dejar de ver a familiares o amigos. Según un reciente estudio, el miedo a que se contagie un familiar se da en casi la mitad de la población estudiada y el temor a perder a un familiar, como consecuencia de la pandemia, se da en más del $40 \%$ de los participantes (Sandín et al., 2020).

Respecto a la estrategia gradual por fases para enfrentar la pandemia según la situación sanitaria de las distintas localidades de Chile, los resultados arrojaron que las personas que tuvieron que permanecer en confinamiento total obligatorio, presentaron más ansiedad ante el desconfinamiento que aquellas personas que no se encontraban en cuarentena. De acuerdo a la definición del constructo, este resultado es esperable, puesto que la ansidad por desconfinamiento debiesen padecerla aquellos que están confinados y no aquellos que pueden circular con mayor libertad por su ciudad, por lo que este resultado es un indicador más de la validez de cosntructo de la EAD. En suma, la ansiedad por desconfinamiento afecta específicamente a quienes han permanecido encerrados ante la inminencia de un desconfinamiento, lo que es particular o especial para este tipo de ansiedad y que lo hace diferente de otras manifestaciones de malestar.

Este estudio presenta algunas limitaciones. Una de ellas, es que el muestreo fue por accesibilidad por lo que no necesariamente los resultados son generalizables para toda la 
población. Por ejemplo, debido a las enormes dificultades que existen para aplicar encuestas presenciales, toda la población que se encuentra sin conexión regular a internet, la que probablemente representa a personas en condiciones de vida de mayor vulnerabilidad socioeconómica, han quedado excluidos del presente estudio. Otra de las limitaciones es que no se consultó por algún diagnóstico psiquiátrico previo, el que podría ser considerado un factor de riesgo y podría afectar la salud mental y respuestas ante el desconfinamiento.

Se sugiere para futuros estudios evaluar la estabilidad temporal del instrumento, su relación con otros constructos psicológicas como las estrategias de afrontamiento y la regulación emocional, su relación con otras variables relevantes que pueden afectar la salud mental durante la pandemia por COVID-19, como la sobrecarga laboral, la interacción trabajo-familia y las clases online, entre otras.

La pandemia del coronavirus COVID-19, no tiene precedentes en la historia reciente, con impactos globales mundiales que incluyen altas tasas de mortalidad, pérdida de ingresos, trabajos, estudios y aislamiento, entre otros. Se desconoce el efecto que tendrá esta crisis en la salud mental, tanto a corto como a largo plazo. Por lo tanto, es relevante contar con instrumentos válidos y confiables que permitan prevenir y contribuir a la salud mental de la población. Esperamos que este instrumento sea un aporte en esa dirección.

\section{Referencias}

Andrades, M., García, F., Concha, P., Valiente, C., \& Lucero, C. (2021). Predictores de síntomas de ansiedad, depresión y estrés a partir del brote epidémico de COVID-19. Journal of Psychopathology and Clinical Psychology, 26(1), 13-22. https://doi.org/10.5944/rppc.26200

Antúnez, Z., \& Vinet, E. V. (2013). Problemas de salud mental en estudiantes de una universidad regional chilena. Revista Médica de Chile, 141(2), 209-216. https://doi.org/10.4067/s003498872013000200010
Arias, P., \& García, F. (2018). Propiedades psicométricas de la Escala de Satisfacción con la Vida en población ecuatoriana adulta. Pensamiento Psicológico, 16(2), 21-29. https://doi.org/10.11144/Javerianacali.PPSI16 -2.ppes

Balaratnasingam, S., \& Janca, A. (2006). Mass hysteria revisited. Current Opinion in Psychiatry, 19(2), 171-174.

https://doi.org/10.1097/01.yco.0000214343.59 $872.7 \mathrm{a}$

Barlow, D. H., Allen, L., \& Choate, M. (2004). Toward a unified treatment for emotional disorders. Behaviour Therapy, 35(2), 205-230. https://doi.org/10.1016/S00057894(04)80036-4

Brooks, S. K., Webster, R. K., Smith, L. E., Woodland, L., Wessely, S., Greenberg, N., \& Rubin, G. J. (2020). The psychological impact of quarantine and how to reduce it: Rapid review of the evidence. The Lancet, (395), 912-920.

https://doi.org/10.1016/S0140-

6736(20)30460-8

Ceberio, M. R. (2020). Psicólogos en el frente: La atención durante la crisis del Covid-19. De las emociones tóxicas a la salud psicológica. Archivos de Medicina (Manizales), 21(1), 225-237.

https://doi.org/10.30554/archmed.21.1.3941.2021

Colegio Oficial de la Psicología de Madrid (2020a). "Síndrome de la cabaña": Cuando la libertad se convierte en un problema. COP. Disponible

en: https://www.copmadrid.org/wp/sindrome-dela-cabana-cuando-la-libertad-se-convierte-enun-problema/

Colegio Oficial de la Psicología de Madrid (2020b). Guía para un afrontamiento psicológico eficaz del proceso de desconfinamiento. COP. Disponible en: https://www.copmadrid.org/web/img_db/publi caciones/guia-para-un-afrontamientopsicologico-eficaz-del-proceso-dedesconfinamiento-y-ahora-que5ec65a193e329.pdf

Da, B. L., Im, G. Y., \& Schiano, T. D. (2020). Coronavirus disease 2019 Hangover: A rising tide of alcohol use disorder and alcohol- 
associated liver disease. Hepatology, 72(3), 1102-1108. https://doi.org/10.1002/hep.31307

Diener, E., Emmps, R., Larsen, R., \& Griffin, S. (1985). The Satisfaction with Life Scale. Journal of Personality Assessment, 49(1), 7175. https://doi.org/10.1207/s15327752jpa4901_13

Ehrlich, H., McKenney, M., \& Elkbuli, A. (2020). Protecting our healthcare workers during the COVID-19 pandemic. The American Journal of Emergency Medicine, 38(7), 1527-1528. https://doi.org/10.1016/j.ajem.2020.04.024

Ferrer, R. (2020). Pandemia por COVID-19: El mayor reto de la historia del intensivismo. Medicina Intensiva, 44(6), 323-324. https://doi.org/10.1016/j.medin.2020.04.002

Galea, S., Merchant, R., \& Lurie, N. (2020). The mental health consequences of COVID-19 and physical distancingthe need for prevention and early intervention. JAMA Internal Medicine, 180(6), 817-818. https://doi:10.1001/jamainternmed.2020.1562

Gautam, R., \& Sharma, M. (2020). 2019-nCoV pandemic: A disruptive and stressful atmosphere for Indian academic fraternity. Brain, Behavior and Immunity, 88 , 948-949.

https://doi.org/10.1016/j.bbi.2020.04.025

Gil, E., González, J. R., \& Meneses, C. (2010). Percepción del riesgo y dinámicas promotoras de salud en adolescentes: Una mirada de género. Revista Española de Drogodependencias, 35, 297-308.

Gobierno de Chile (2020). Paso a paso nos cuidamos. https://www.gob.cl/pasoapaso/

Hair, J. F. Jr., Black, W. C., Babin, B. J., \& Anderson, R. E.( 2010). Multivariate data analysis (7th ed.). Prentice Hall.

Intrieri, E., Dotta, G., Fontanelli, K., Bianchini, C., Bardi, F., Campatelli, F., \& Casagli, N. (2020). Operational framework for flood risk communication. International Journal of Disaster Risk Reduction, 46, 1-9. https://doi.org/10.1016/j.ijdrr.2020.101510

Lee, E. (2020). Coronavirus Anxiety Scale: A brief mental health screener for COVID-19 related anxiety. Death Studies, 44, 393-401. https://doi.org/10.1080/07481187.2020.1748481

Li, Z., Ge, J., Yang, M., Feng, J., Qiao, M., Jiang, R.,... Yang, C. (2020). Vicarious traumatization in the general public, members, and non-members of medical teams aiding in COVID-19 control. Brain, Behavior, and Immunity, 88, 916-919.

https://doi.org/10.1016/j.bbi.2020.03.007

Lovibond, S., \& Lovibond P. (1995). Manual for the Depression Anxiety Stress Scales. Psychology Foundation of Australia.

Marjanovic, Z., Greenglass, E., \& Coffey, S. (2007). The relevance of psychosocial variables and working conditions in predicting nurses' coping strategies during the SARS crisis: An online questionnaire survey. International Journal of Nursing Studies, 44(6), 991-998.

https://doi.org/10.1016/j.ijnurstu.2006.02.012

Martínez-Munguía, C., Quintana-Rodríguez, C., \& Ortiz-Rueda, G. (2014). Género y conductas de riesgo para la salud: Una aproximación desde un punto de vista interconductual. Journal of Behavior, Health \& Social Issues. 6(2), 57-69 https://doi.org/10.5460/jbhsi.v6.2.41614

Ministerio de Salud, Chile (2020). Síndrome de la Cabaña: El temor a salir post cuarentena. Minsal. Disponible en: https://www.saludohiggins.cl/sindrome-de-lacabana-el-temor-a-salir-post-cuarentena/

Mora-Rodríguez, A., \& Melero-López, I. (2021). Seguimiento informativo y percepción del riesgo ante la Covid-19 en España. Comunicar: Revista Científica de Comunicación y Educación, 29(66), 71-81. https://doi.org/10.3916/c66-2021-06

O'Connor, B. P. (2000). SPSS and SAS programs for determining the number of components using parallel analysis and Velicer's MAP test. Behavior Research Methods, Instruments, \& Computers, 32(3), 396-402. https://doi.org/10.3758/BF03200807

Organización Mundial de la Salud (2020). Brote de enfermedad por coronavirus (COVID-19). Disponible en: https://www.who.int/es/emergencies/diseases/ novel-coronavirus-2019

Organización Mundial de la Salud (2020). Consideraciones relativas a los ajustes de las medidas de salud pública y sociales en el contexto de la COVID-19. Disponible en: https://apps.who.int/iris/bitstream/handle/1066 
5/331970/WHO-2019-nCoV-

Adjusting_PH_measures-2020.1-spa.pdf

Prol, S. M., \& Silva, J. S. (2020). El Síndrome de la Cabaña como metáfora del SER. Revista APRA, $1, \quad 1-11$ https://www.apra.org.ar/pdf/junio_2020/ElSi $\%$ CC\%81ndromedelaCaban\%CC\%83acomo meta\%CC \%81foradelSER.pdf

Reynolds D., Garay J., Deamond S., Moran M., Gold, W., \& Styra, R. (2008). Understanding, compliance and psychological impact of the SARS quarantine experience. Epidemiology and Infection, 136(7), 997-1007. https://doi.org/10.1017/S0950268807009156

Rosenblatt, P., Anderson, R., \& Johnson. P. (1984) The meaning of "Cabin Fever". The Journal of Social Psychology, 123(1), 43-53. https://doi.org/10.1080/00224545.1984.99245 12

Salari, N., Far, A., Jalali, R., Vaisi-Raygani, A., Rasoulpoor, S., Mohammadi, M., Rasoulpoor, S., \& Khaledi-Paveh, B. (2020). Prevalence of stress, anxiety, depression among the general population during the COVID-19 pandemic: A systematic review and meta-analysis. Globalization and Health, 16, 1-11. https://doi.org/10.1186/s12992-020-00589-w

Sandín, B., Valiente, R., García-Escalera, J., \& Chorot, R. (2020). Impacto psicológico de la pandemia de COVID-19: Efectos negativos y positivos en población española asociados al periodo de confinamiento nacional. Revista de Psicopatología y Psicología Clínica, 25(1), 1-22. https://doi.org/10.5944/rppc.27569

Santabárbara, J., Lasheras, I., Lipnicki, D., Bueno-Notivol J., Pérez-Moreno M., LópezAntón R., De la Cámara C., Lobo A., \& Gracia-García P. (2021). Prevalence of anxiety in the COVID-19 pandemic: An updated meta-analysis of community-based studies. Progress in NeuroPsychopharmacology and Biological Psychiatry, 109(13), 1-13. https://doi: 10.1016/j.pnpbp.2020.110207

Saravia, M., Cazorla, P., \& Cedillo, L. (2020). Nivel de ansiedad de estudiantes de medicina de primer año de una universidad privada del Perú en tiempos de COVID-19. Revista de la Facultad de Medicina Humana, 20(4), 568-
573.

http://dx.doi.org/10.25176/rfmh.v20i4.3198

Sciarrino, N. A., Myers, U. S., \& Wangelin, B. C. (2020). When chaos is the norm: How some veterans with PTSD are continuing to engage in trauma-focused treatments during the COVID-19 pandemic. Psychological Trauma: Theory, Research, Practice, and Policy, 12(S1), S69-S70. http://dx.doi.org/10.1037/tra0000718

Sepúlveda, B. (19 de julio de 2020). Desescalada emocional y "síndrome de la cabaña": el alto precio por salir de la cuarentena. La Tercera. Disponible en: https://www.latercera.com/quepasa/noticia/desescalada-emocional-ysindrome-de-la-cabana-el-alto-precio-porsalir-de-lacuarentena/IRN2ZOY7K5DSPI4JL2WMWPJ ZUI/

Silva, M. (2020). Vulnerabilidades da população idosa durante a pandemia pelo novo coronavírus. Revista Brasileira de Geriatria e Gerontologia, 23(3), e200319. https://doi.org/10.1590/198122562020023.200319

Talevi, D., Socci, V., Carai, M., Carnaghi, G., Faleri, S., Trebbi, E., ... \& Pacitti, F. (2020). Mental health outcomes of the CoViD-19 pandemic. Rivista di Psichiatria, 55(3), 137144. https://doi.org/10.1708/3382.33569

Tan, W., Hao, F., McIntyred, S., Jiang, L., \& Jiang, X. (2020). Is returning to work during the COVID-19 pandemic stressful? A study on immediate mental health status and psychoneuroimmunity prevention measures of Chinese workforce. Brain, Behaviour and Immunity. 87, 84-92.

https://doi.org/10.1016/j.bbi.2020.04.055

Valdés, A. (2020). Síndrome de la cabaña: 6 Enfermedad real? Recuperado desde: https://www.hsnstore.com/blog/salud-ybelleza/buenos-habitos/sindrome-de-lacabana-enfermedad-real/

Vindegaard, N., \& Benros, M. E. (2020). COVID19 pandemic and mental health consequences: Systematic review of the current evidence. Brain, Behavior, and Immunity, 89, 531-542. https://doi.org/10.1016/j.bbi.2020.05.048 
Wang, C., Pan, R., Wan, X., Tan, Y., Xu, L., Ho, C. S., \& Ho, R. C. (2020). Immediate psychological responses and associated factors during the initial stage of the 2019 coronavirus disease (COVID-19) epidemic among the general population in China. International Journal of Environmental Research and Public Health (IJERPH), 17, 17-29.

https://doi.org/10.3390/ijerph17051729

Wolf, I., Waissengrin, B., \& Pelles, S. (2020). Breaking bad news via telemedicine: A new challenge at times of an epidemic. The Oncologist, 25(6), e879-e880.

https://doi.org/10.1634/theoncologist.20200284

Xiang Y.T., Yang Y., Li, W., Zhang, L., Zhang, Q., \& Cheung, T. (2020). Timely mental health care for the 2019 novel coronavirus outbreak is urgently needed. The Lancet Psychiatry, 7, 228-229.

https://doi.org/10.1016/s2215-0366(20)300468 